\title{
Single-Kernel Near-Infrared Analysis for Evaluating Wheat Samples for Fusarium Head Blight Resistance
}

\author{
Kamaranga H. S. Peiris, ${ }^{1}$ Yanhong Dong, ${ }^{2}$ William W. Bockus, ${ }^{3}$ and Floyd E. Dowell ${ }^{4,5}$
}

\section{ABSTRACT}

Cereal Chem. 91(1):35-40

This report describes a method to estimate the bulk deoxynivalenol (DON) content of wheat grain samples with the single-kernel DON levels estimated by a single-kernel near-infrared (SKNIR) system combined with single-kernel weights. The described method estimated the bulk DON levels in $90 \%$ of 160 grain samples to within $6.7 \mathrm{ppm}$ of DON when compared with the DON content determined with the gas chromatography-mass spectrometry method. The single-kernel DON analysis showed that the DON content among DON-containing kernels (DCKs) varied considerably. The analysis of the distribution of DON levels among all kernels and among the DCKs of grain samples is helpful for the in-depth evaluation of the effect of varieties or fungicides on Fusarium head blight (FHB) reactions. The SKNIR DON analysis and estimation of the single-kernel DON distribution patterns demonstrated in this study may be helpful for wheat breeders to evaluate the FHB resistance of varieties in relation to their resistance to the spread of the disease and resistance to DON accumulation.
Fusarium head blight (FHB) of wheat is a destructive disease caused by Fusarium graminearum and several other Fusarium species (Parry et al 1995; McMullen et al 1997; Miedaner 1997; Dill-Macky 2010). In addition to significant losses in grain yield and quality, the trichothecene mycotoxins such as deoxynivalenol (DON), produced by the Fusarium fungus in infected wheat kernels, are a serious problem affecting grain marketability and the safety of food and feed (Johnson et al 2001; Finamore et al 2004). In terms of both economic and environmental points of view, host resistance has long been considered the most practical and effective means of controlling FHB; such host resistance to FHB in wheat is the result of several morphological and physiological resistance mechanisms (Schroeder and Christensen 1963; Mesterhazy 1995).

In wheat breeding programs, it is necessary to evaluate numerous wheat varieties for FHB resistance, and the assessment of FHB resistance is based on the evaluation of such disease parameters as the disease incidence, severity, percentage of Fusariumdamaged kernels (FDKs), and DON levels in grains. In addition, certain weighted indexes, including the FHB, ISK (incidence, severity, and kernel damage), and DISK (DON, incidence, severity, and kernel damage) indexes, based on some of the above disease parameters, are also used (Gilbert and Woods 2006; Thompson 2010). The ISK index uses a weighted mean with weights of $0.3,0.3$, and 0.4 for the incidence, severity, and kernel damage measured as the $\%$ FDK, respectively, where ISK $=[0.3 \times$ incidence $(\%)]+[0.3 \times$ severity $(\%)]+[0.4 \times$ FDK $(\%)]$. DISK uses a weighted mean with weights of $0.2,0.2,0.3$, and 0.3 for DON, incidence, severity, and kernel damage, respectively, where DISK $=[0.3 \times$ DON $(\mathrm{ppm})]+[0.2 \times$ incidence $(\%)]+[0.2 \times$ severity $(\%)]+[0.3 \times$ FDK $(\%)]$.

The FDK and DON levels in harvested grains are two important parameters often used to assess the FHB reaction of wheat varieties and to assess the efficacy of fungicides to control FHB. FDKs are usually visually estimated by trained personnel through

\footnotetext{
${ }^{1}$ Department of Biological and Agricultural Engineering, Kansas State University, Manhattan, KS, U.S.A.

${ }^{2}$ Department of Plant Pathology, University of Minnesota, St. Paul, MN, U.S.A.

${ }^{3}$ Department of Plant Pathology, Kansas State University, Manhattan, KS, U.S.A.

${ }^{4}$ USDA, ARS, CGAHR, Engineering and Wind Erosion Research Unit, Manhattan,

KS, U.S.A. The mention of trade names or commercial products in this publication is solely for the purpose of providing specific information and does not imply recommendation or endorsement by the U.S. Department of Agriculture. USDA is an equal opportunity provider and employer.

${ }^{5}$ Corresponding author. Phone: (785) 776-2753. E-mail: floyd.dowell@ars.usda.gov
}

http://dx.doi.org/10.1094/CCHEM-11-12-0157-R

This article is in the public domain and not copyrightable. It may be freely reprinted with customary crediting of the source. AACC International, Inc., 2014. the manual sorting and counting of sound kernels and FDKs. Bulk or single-kernel DON levels are determined with standard laboratory methods such as gas chromatography-mass spectrometry (GC-MS) and high-performance liquid chromatography (Mirocha et al 1998; Koch 2004; Jiang et al 2006). However, the manual sorting of FDKs is laborious and is subject to inconsistencies because of intra-rater repeatability and inter-rater reliability, and laboratory techniques for determining DON levels are time consuming, expensive, and destructive.

An automated single-kernel near-infrared (SKNIR) sorter has been developed for the rapid, nondestructive, and objective evaluation and sorting of single wheat kernels for a variety of quality traits (Dowell et al 2006). Wegulo and Dowell (2008) compared the capability of this SKNIR system to estimate FDKs with the visual estimates assigned by experienced raters. Visual sorting was found to be strongly correlated with SKNIR sorting; furthermore, the SKNIR system had a wider range of Fusarium-damaged kernel detection and was more consistent.

Peiris et al (2010) used the SKNIR system to classify visually sound kernels and FDKs with an accuracy of 98.8 and $99.9 \%$, respectively. The sound fraction had no or very little accumulation of DON, whereas the FDK fraction was sorted into two-to-three fractions with low to high DON levels, as confirmed by analyzing the DON levels of the sorted fractions with a standard laboratory method. Indeed, the sorting of grains into several fractions depending on their DON levels provides more information compared with techniques that estimate the DON concentration of unsorted bulk samples. Thus, varieties that exhibit high DON levels resulting from a small fraction of kernels with high DON contents can be delineated from those that exhibit low DON contents because of a high proportion of kernels with low DON levels, allowing the identification of varieties that can produce a high proportion of sound and low-DON kernels, regardless of the bulk DON content.

Single-kernel analysis of the DON levels in a bulk grain sample can offer more information on the distribution of DON levels among the kernels in a grain sample, perhaps providing an indication of the underlying FHB resistance mechanisms. Here, we describe a SKNIR spectroscopic method to estimate the bulk DON content and analyze the single-kernel DON distribution among kernels in grain samples for the comprehensive evaluation and comparison of the effect of varieties and fungicides on FHB reactions.

\section{MATERIALS AND METHODS}

Description of Samples. The grain samples for this study were selected from scab nursery trials grown in 2009-2010 (2010) and 
2010-2011 (2011) under heavy disease pressure to test several wheat cultivars and fungicides for FHB control. These grain samples consisted of wheat cultivars Art, Everest, Fuller, Heyne, Karl 92, Overley, Roane, Santa Fe, Truman, and Tomahawk grown with or without Prosaro fungicide applications. Only eight cultivars were evaluated in each year; cultivars Roane and Truman in the 2010 trial were replaced by cultivars Art and Santa Fe in 2011. Prosaro 421 SC fungicide was applied at $6.5 \mathrm{fl} \mathrm{oz} / \mathrm{ac}$ at the fully headed growth stage. Nested within this experiment, Caramba, Prolene, Topguard, and Folicar fungicides were also tested with application rates of 13.5, 5.0, 14.0, and $4.0 \mathrm{fl} \mathrm{oz} / \mathrm{ac}$, respectively, using cultivar Fuller. Each of these 20 cultivar $\times$ fungicide treatment combinations had four replicate plots in the field. The disease pressure was achieved by applying a corn spawn inoculum of the Z-3639 (NRRL 29214) isolate of F. graminearum to the soil $\left(65 \mathrm{~g} / \mathrm{m}^{2}\right)$. The spawn produced fungal perithecia, which released ascospores during wheat heading. The infections were enhanced by running overhead impulse sprinklers for 3 min every hour from 9:00 p.m. through 6:00 a.m. during the grain-filling stage. The plots were harvested with a plot combine. All of the 160 grain samples were used for the development of the method for estimation of the bulk DON content of grain samples. The distribution of the single-kernel DON levels in wheat cultivars grown with or without Prosaro fungicide was demonstrated using two selected wheat cultivars (moderately FHB-resistant Everest and FHB-susceptible Tomahawk).

DON Predictions. A subsample of approximately $100 \mathrm{~g}$ was drawn from the harvested grains of each plot and cleaned to remove the chaff and other debris in addition to scabby kernels. Approximately $50 \mathrm{~g}$ of kernels from the cleaned sample was

TABLE I

Deoxynivalenol (DON) Concentration Determined by the Single-Kernel NIR Method (SK DON) and Standard GC-MS Method (Lab DON) ${ }^{\mathrm{a}}$

\begin{tabular}{llcrr}
\hline Year & Cultivar & Fungicide & \multicolumn{1}{c}{$\begin{array}{c}\text { SK DON } \\
(\mathbf{p p m})\end{array}$} & \multicolumn{1}{c}{$\begin{array}{c}\text { Lab DON } \\
(\mathbf{p p m})\end{array}$} \\
\hline 2010 & Everest & $\ldots$ & $6.3 \pm 1.1$ & $3.5 \pm 0.4$ \\
& Tomahawk & $\ldots$ & $29.8 \pm 5.7$ & $13.9 \pm 1.4$ \\
& Everest & Prosaro & $5.5 \pm 1.0$ & $3.8 \pm 0.3$ \\
& Tomahawk & Prosaro & $17.7 \pm 2.6$ & $11.8 \pm 0.5$ \\
2011 & Everest & $\ldots$ & $1.5 \pm 1.2$ & $1.8 \pm 0.2$ \\
& Tomahawk & $\ldots$ & $3.8 \pm 0.7$ & $9.4 \pm 1.1$ \\
& Everest & Prosaro & $0.6 \pm 0.3$ & $2.0 \pm 0.3$ \\
& Tomahawk & Prosaro & $1.8 \pm 0.2$ & $6.2 \pm 0.4$ \\
\hline
\end{tabular}

${ }^{a}$ Wheat was grown with or without the application of fungicide. The DON values are the mean of four 500-kernel samples \pm standard error.

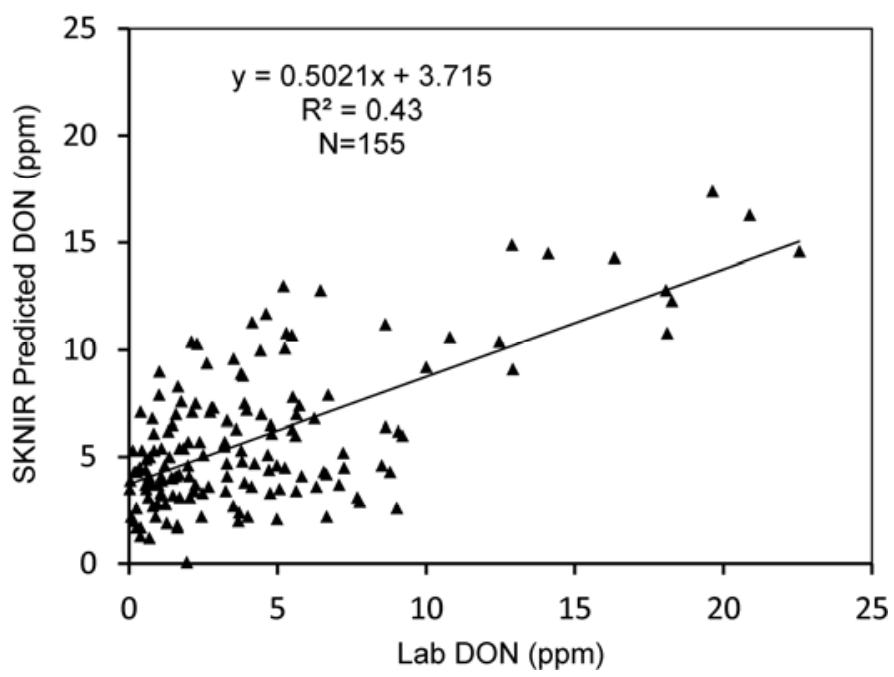

Fig. 1. Relationship between the single-kernel NIR-estimated bulk deoxynivalenol (DON) level and DON determined by the standard GC-MS method. loaded into the SKNIR grain feeder, which was programmed to estimate the single-kernel DON levels in 500 kernels using the calibration developed to estimate the DON concentration in single wheat kernels (Peiris et al 2010). This single-kernel DON calibration $\left(R^{2}=0.72\right.$; standard error of cross-validation $=154.2 \mathrm{ppm}$ with 5 PLS factors) could successfully separate kernels with low DON concentration $(<60 \mathrm{ppm})$ from kernels with high DON concentration (>60 ppm) with 95.7 and $96.7 \%$ accuracy, respectively. Single-kernel DON levels of the high-DON fraction (>60 ppm) were estimated with $R^{2}=0.87$ and a standard error of prediction (SEP) of $60.8 \mathrm{ppm}$.

The 500 kernels were sorted into four groups based on the estimated DON value: kernels with nondetectable DON levels were sorted into bin 1, whereas those kernels having $<60$ ppm, 60-160 ppm, and $>160$ ppm estimated DON were sorted into bins 2, 3, and 4, respectively. The kernels sorted into bins 2-4 were regarded as DON-containing kernels (DCKs). The number of kernels and total weight of kernels sorted into each bin were recorded. The SKNIR-estimated bulk DON level of the 500-kernel samples was determined with the following equation:

$$
\operatorname{DON}(\mathrm{ppm})=\sum \frac{\operatorname{SKDON}(\mathrm{ppm}) \times \mathrm{SKW}(\mathrm{g})}{\mathrm{TKW}(\mathrm{g})}
$$

where SKDON is the SKNIR-predicted single-kernel DON value, SKW is the single-kernel weight, and TKW is the total kernel weight of the 500 kernels from each grain sample. The average weight of the kernels in each bin was used as the single-kernel weight for all the kernels in the respective bin. To evaluate the performance of this SKNIR bulk DON estimation method, the SKNIR-estimated bulk DON values of 160 wheat kernel samples representing the 10 wheat cultivars were compared with the DON values of representative samples estimated with a GC-MS laboratory method.

Two-sample $t$ tests assuming unequal variances were performed on the \% DCK and average DON values of grain samples of cultivars Everest and Tomahawk grown with or without fungicide applications in 2010 and 2011 to test the statistical significance of the mean DON level differences between the two cultivars, growing seasons, and fungicide treatment within the cultivars.

Estimates of Sampling Error. Using the same sort setting as described earlier, another 3,000 kernels were sorted from a sample that had a DON value of $8.0 \mathrm{ppm}$ as determined by GC-MS. From these 3,000 kernels, 500-kernel samples were randomly selected 60 times, and the \% DCK and bulk DON values of those

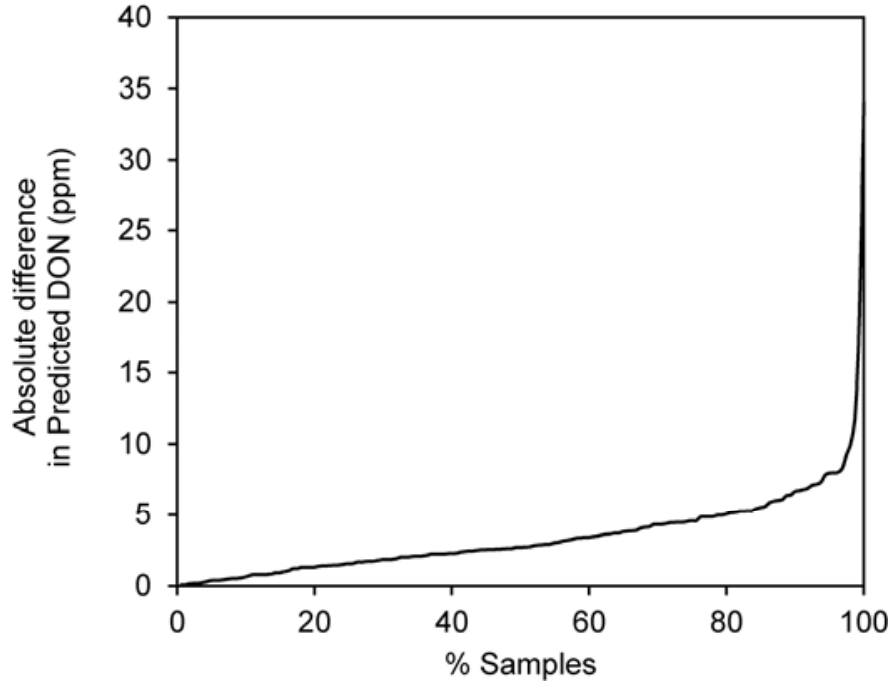

Fig. 2. The absolute difference between the single-kernel NIR deoxynivalenol (DON) and GC-MS-determined DON levels of 160 grain samples. 
samples were computed to estimate the sampling error associated when using 500-kernel samples for DON estimation.

DON Distributions. The distribution of the SKNIR-estimated single-kernel DON levels between the cultivars and fungicide treatments was also compared. The single-kernel DON distribution among the kernels in the grain samples was compared by plotting the DON content against percentage of kernels. A total of 100 DCKs were randomly selected from Everest and Tomahawk cultivars grown without fungicide application, and the DON contents of these selected DCKs were sorted from high to low and plotted against the kernel number to demonstrate the comparison of the DON distribution among the DCKs of the two cultivars.

Cultivars Everest and Roane, which had the same bulk DON (4.7 ppm) and DCK (6.0\%) levels, were used to demonstrate the different modes of bulk DON accumulation in relation to the single-kernel DON distribution pattern between the two grain samples. The kernel weight and DON level data of the 500-kernel samples were sorted from the lowest to highest DON levels. Starting from the sound kernels with no detectable DON, the bulk DON values were estimated using the cumulative weight and cumulative DON content as each DCK with the lowest to highest DON content was added.

\section{RESULTS AND DISCUSSION}

SKNIR Method for the Estimation of Bulk DON. The bulk DON values of wheat cultivars Everest and Tomahawk grown with or without fungicide in two growing seasons are presented in Table I. Overall, the DON values determined with the standard laboratory method were higher in $2010(8.3 \mathrm{ppm})$ than in 2011 $(4.8 \mathrm{ppm})(P$ value $=0.0162)$. The susceptible cultivar Tomahawk had a higher overall DON value of $10.3 \mathrm{ppm}$ compared with a mean DON content of $2.8 \mathrm{ppm}$ in Everest $(P$ value $<0.0001)$. Fungicide application reduced the mean DON content in the Tomahawk grains from 11.7 to $9.0 \mathrm{ppm}$; however, the reduction in the DON content was only significant at $\alpha=0.10(P$ value $=0.0604)$. There was no significant difference in the mean DON levels of Everest with regard to the fungicide treatment $(P$ value $=0.3038)$. This pattern of DON variation between the years, cultivars, and fungicide treatments was the same when the sample DON levels were estimated with the SKNIR method. The high DON content in 2010 may be because of the prevalence of more favorable climatic conditions for the spread of the disease. In Kansas, severe FHB occurrence was reported in 2010, although the FHB losses were very low in 2011 (Lilleboe 2010, 2011). The Everest cultivar is more resistant to FHB compared with Tomahawk, as shown by the lower DON levels when grown under the same conditions.

The feasibility of the SKNIR method to estimate the bulk DON content was further evaluated by comparing the SKNIR-estimated DON content in 160 bulk kernel samples with the DON levels of representative subsamples determined with the standard GC-MS method. The SKNIR method predicted the bulk DON content with a root mean squared error of prediction $($ RMSEP $)=4.98$ ppm, bias $=-1.04 \mathrm{ppm}$, and $R^{2}=0.35(P<0.0001)$. The removal of five outliers reduced RMSEP to $3.69 \mathrm{ppm}$ with bias $=-1.62$ ppm and $R^{2}=0.43(P<0.0001)$ (Fig. 1). The absolute difference of the SKNIR-estimated and lab-determined DON values of 160 samples were plotted against the percentage of samples. The SKNIR method estimated the DON levels of $90 \%$ of the samples within 6.7 ppm of DON (Fig. 2).

Sampling error is a reason for the observed differences between the SKNIR-estimated and lab-determined bulk DON contents. To assess the sampling error in the SKNIR bulk DON estimation when using 500 kernels per sample, 3,000 kernels from a sample with a lab-determined DON value of $8.0 \mathrm{ppm}$ were sorted; from this, 50 random 500-kernel samples were selected, and the DON values were computed. The estimated DON values ranged from 5.4 to $15.8 \mathrm{ppm}$, with a mean of $9.7 \mathrm{ppm}$ and standard deviation of $1.7 \mathrm{ppm}$. The 3,000-kernel sample had $13.7 \%$ DCKs, whereas the DCKs in the 50 randomly selected 500-kernel samples varied from 10.6 to $16.4 \%$. When the grain samples with low FHB damage were used for the analysis, the probability that the \% FDK and DON-level distribution within the FDKs was exactly represented in the 500-kernel sample was low compared with a sample with more extensive FHB damage. This result may be the reason for the high variability observed in the SKNIR-estimated DON levels when the sample DON level is low (Fig. 1); such variability could be expected to be lower if the number of kernels per sample is further increased.
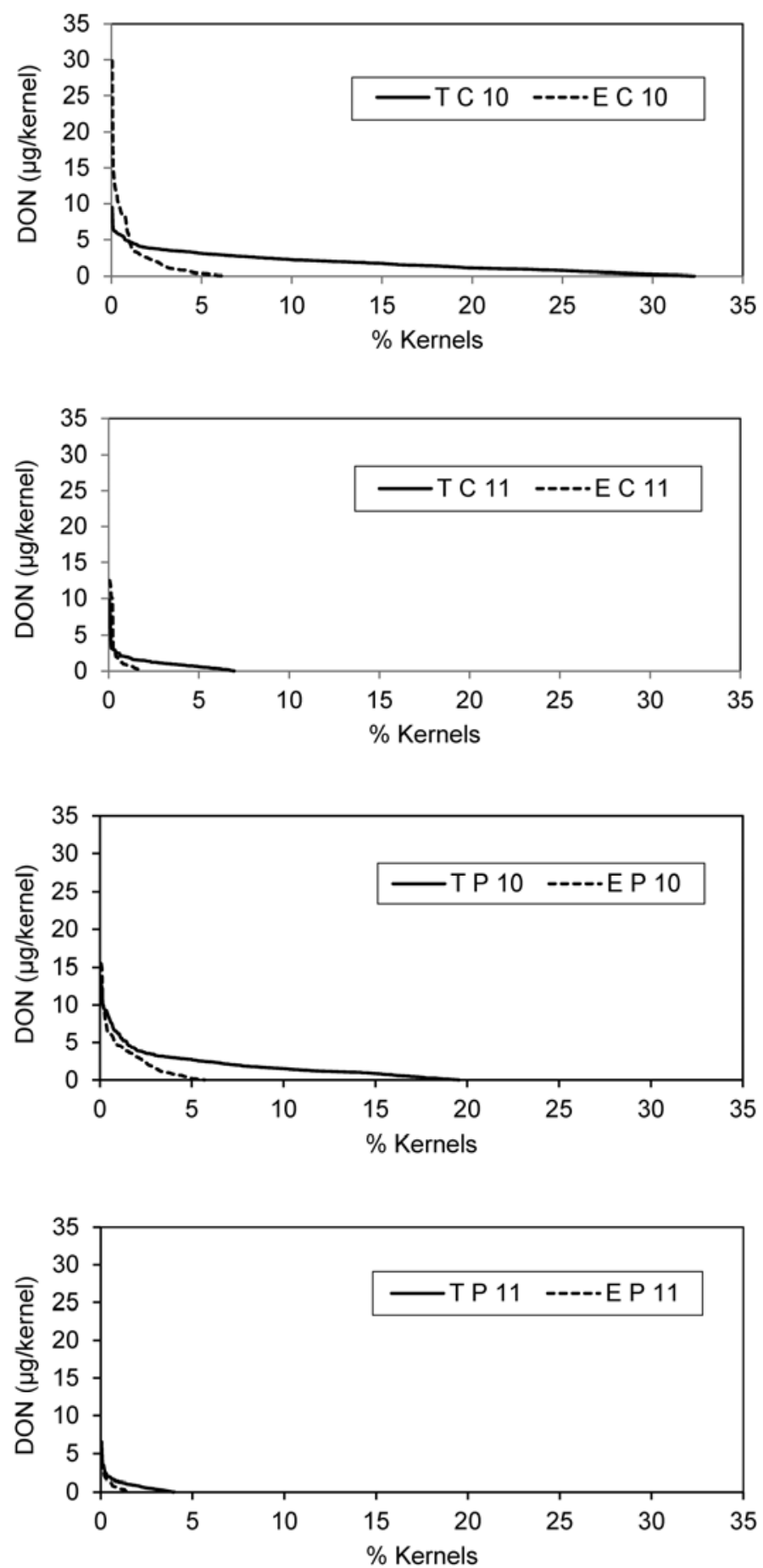

Fig. 3. Single-kernel deoxynivalenol level distribution in the harvested grains of the susceptible cultivar Tomahawk $(\mathrm{T})$ and the resistant cultivar Everest (E) with Prosaro fungicide (P) or without it (C) in the 2010 (10) and 2011 (11) seasons. 
In addition, the use of the average weight of the kernels in each bin as the individual single-kernel weight for the kernels in the respective bins can also add an error to the DON estimation. The SKNIR system does not have the capability of recording the weight of individual kernels as the kernels are sorted. Therefore, the addition of a weighing device for recording single-kernel weights may be helpful to improve the capacity of the system to estimate DON with less error. Therefore, it can be reasonably expected that the use of individual kernel weights and an increase in the sample size may further improve the ability of SKNIR to predict sample DON levels with less error. This possibility warrants further testing.

Distribution of DON Levels Among Single Kernels in Grain Samples. The ability of the SKNIR system to predict DON levels rapidly and nondestructively makes it possible to examine how single-kernel DON levels vary among the kernels within a sample.

The single-kernel DON levels data were sorted from high to low and plotted against the percentage of kernels in the sample. These figures show the DON level of single kernels against kernel count and illustrate DON accumulation patterns as well as DON
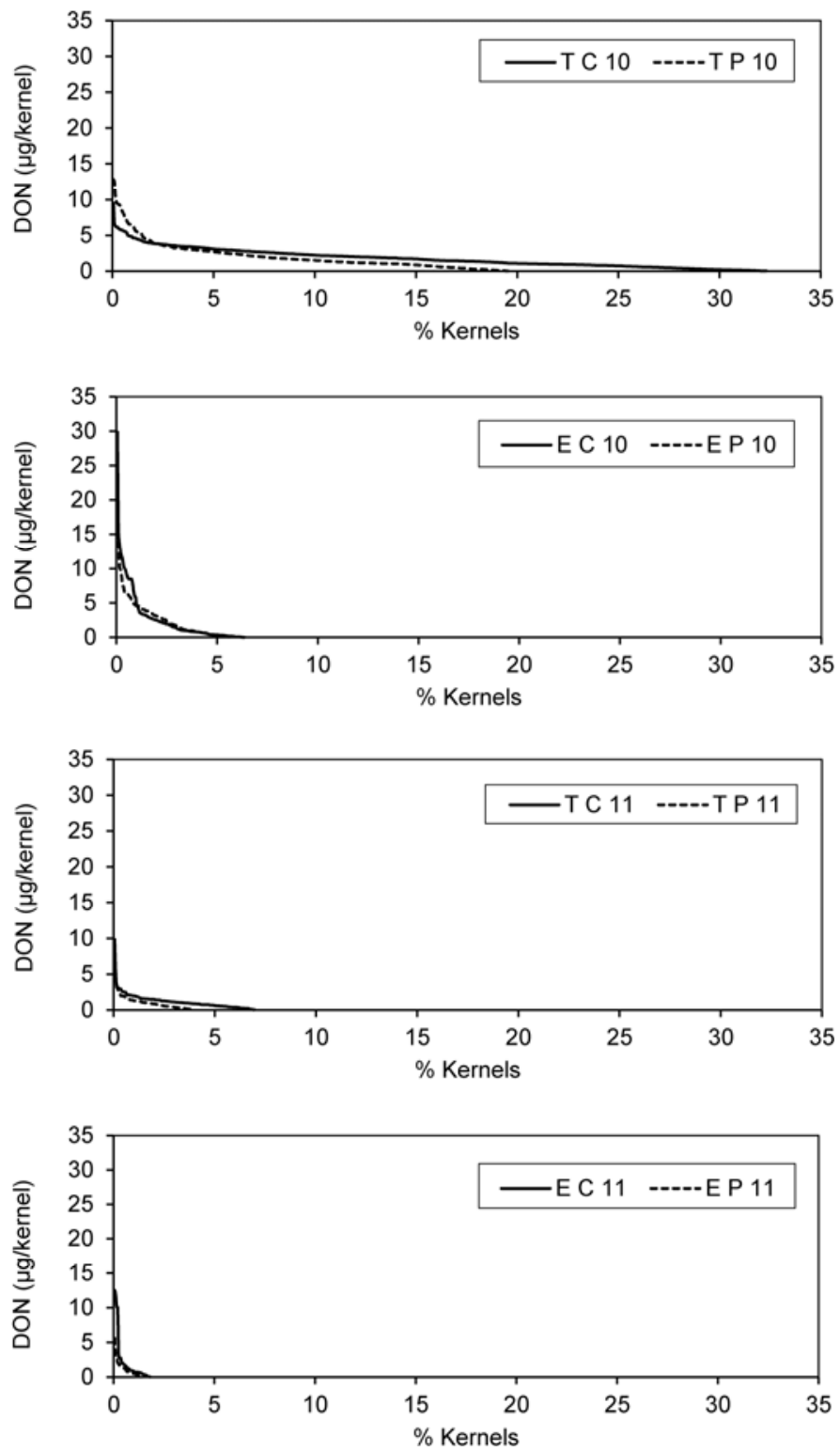

Fig. 4. Single-kernel deoxynivalenol level distribution in the harvested grains of the control $(\mathrm{C})$ and Prosaro fungicide-treated $(\mathrm{P})$ wheat cultivars Tomahawk (T) and Everest (E) in the 2010 (10) and 2011 (11) seasons. spread among kernels in grain samples, which could be used to visualize and compare the effect of cultivars or fungicides on FHB. The effects of the cultivar on the distribution of the singlekernel DON levels of harvested grains when grown with or without fungicide application in two seasons are shown in Figure 3. The overall DON levels and DCKs were lower in 2011 compared with 2010. However, Everest contained a lower percentage of DCKs than Tomahawk in both seasons, regardless of whether the plants were grown with or without fungicide application. In 2010, Everest and Tomahawk had 6.4 and $32.3 \%$ DCKs, which was reduced to 5.7 and $19.6 \%$, respectively, when the plants were grown with fungicide application. A similar pattern of DCKs was observed between the two varieties in 2011, although the overall DCKs were lower. The lower DCK level consistently found in Everest may be because of this cultivar having a higher type II resistance (resistance to fungal spread within the head) compared with Tomahawk.

The effect of fungicide application on the distribution of the single-kernel DON levels of the harvested grains of the two cultivars in the two seasons is shown in Figure 4. The application of fungicide in 2010 significantly reduced the DCKs in Tomahawk from 32.3 to $19.6 \%(P$ value $=0.0294)$, whereas the change in Everest from 6.4 to $5.7 \%$ was not significant $(P$ value $=0.2907)$ (Fig. 4). Fungicide application in 2011 also significantly reduced the DCKs in Tomahawk from 7.0 to $4.0 \%(P$ value $=0.0182)$, whereas the reduction in DCKs from 1.8 to $1.4 \%$ for Everest was not significant $(P$ value $=0.3147)$. These results show that application of fungicide reduced the DON levels in the harvested grains of Tomahawk by reducing the percentage of DCKs. The application of fungicide significantly reduced the DCK levels in the FHB-susceptible cultivar Tomahawk in both years, irrespective of the disease pressure.

These results showed that DON is not equally distributed among all the kernels in a sample. Indeed, only a certain proportion of kernels in an FHB-infected grain sample contain DON. Moreover, the DON levels also vary among DCKs. Thus, the evaluation of the DON distribution among DCKs is also important because varieties that accumulate higher DON levels owing to a small fraction of DCKs that contain higher DON levels, most likely because of an inability of the variety to metabolize and detoxify DON, could be identified.

Distribution of DON Levels Among DCKs. The distribution of the DON content among the DCKs of Tomahawk and Everest was compared by analyzing the distribution of the SKNIR-predicted DON contents in 100 randomly selected DCKs (Fig. 5). DCKs were selected from the harvested grains of the two varieties

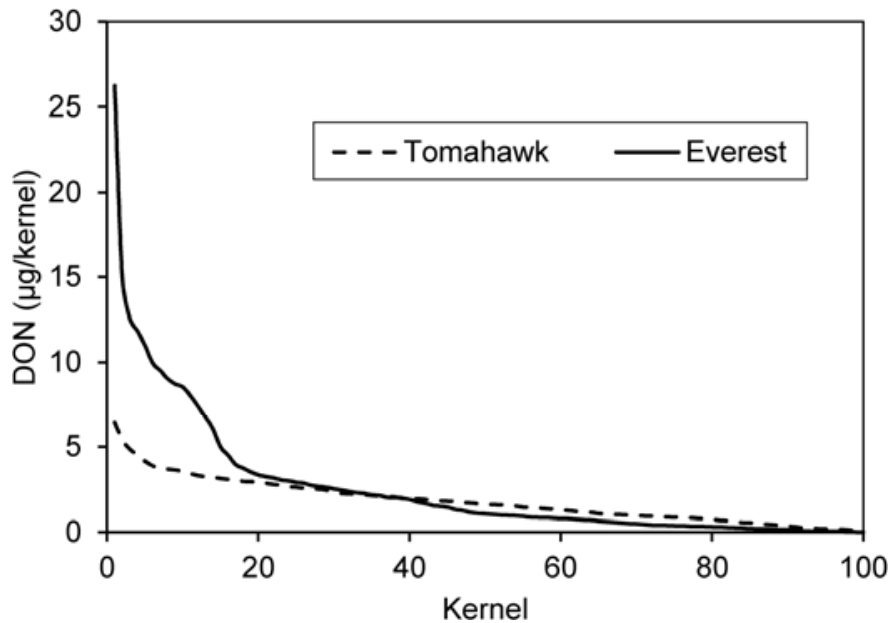

Fig. 5. Distribution of the deoxynivalenol (DON) levels in $100 \mathrm{DON}-$ containing kernels of cultivars Tomahawk and Everest grown in 2010 without fungicide application. 
grown under controlled conditions without fungicide application in 2010, and the pattern of variation of the DON content among the kernels was different for the two varieties. Approximately $80 \%$ of the DCKs of both varieties contained DON contents less than $5 \mu \mathrm{g} / \mathrm{kernel}$. However, the remaining 20\% of Everest DCKs had higher DON levels compared with Tomahawk, which may be because these kernels were from spikelets closer to the points of initial infection. Peiris et al (2011) measured the single-kernel DON levels in the kernels of artificially inoculated spikes of two wheat varieties and showed that the kernels closer to the point of inoculation had higher DON levels, whereas the DON levels gradually decreased in the kernels that were increasingly distal to the point of inoculation. Significant differences were also found in the magnitude of the single-kernel DON levels between the two varieties grown under similar greenhouse environments and inoculated with the same strain of $F$. graminearum, results that were most likely because of differences in mycotoxin detoxification ability. Some studies have indicated that wheat varieties show differences in their ability to detoxify DON (Lemmens et al 2005; Sneller et al 2012), and it appears that Tomahawk may be able to resist DON accumulation better compared with Everest. Additionally, Tomahawk may be more efficient than Everest at detoxifying accumulated DON.

Contribution of DCKs to the Accumulation of Bulk DON in Grain Samples. When the DON content and weight of individual single kernels within a grain sample are known, two wheat varieties having the same DCK and bulk DON contents can be differentiated by examining how the DON levels are distributed among the DCKs and how those DCKs contribute to the accumulation of DON levels in the bulk sample. Using two 500-kernel samples from Everest and Roane varieties, both of which had $4.7 \mathrm{ppm}$ DON and 6.0\% DCKs, we assessed the cumulative bulk DON levels of the samples as influenced by the DCKs with lowest to highest DON contents, as shown in Figure 6. By sorting the DCKs from lowest to highest DON levels, the cumulative bulk DON contents of two samples will increase in two different ways, as shown in Figure 6. This result shows that, if $2 \%$ of the kernels with the highest DON contents were removed from the samples, the bulk DON levels of Everest and Roane would be approximately 1.2 and $1.5 \mathrm{ppm}$, respectively. Similarly, to obtain a final bulk DON value of $1.0 \mathrm{ppm}$ in the harvested grains by removing the kernels with the highest DON content, it would be necessary to remove approximately $2.8 \%$ of the kernels with the highest DON levels from Roane, whereas only approximately $2.4 \%$ of the kernels would need to be removed from the Everest sample. Thus,

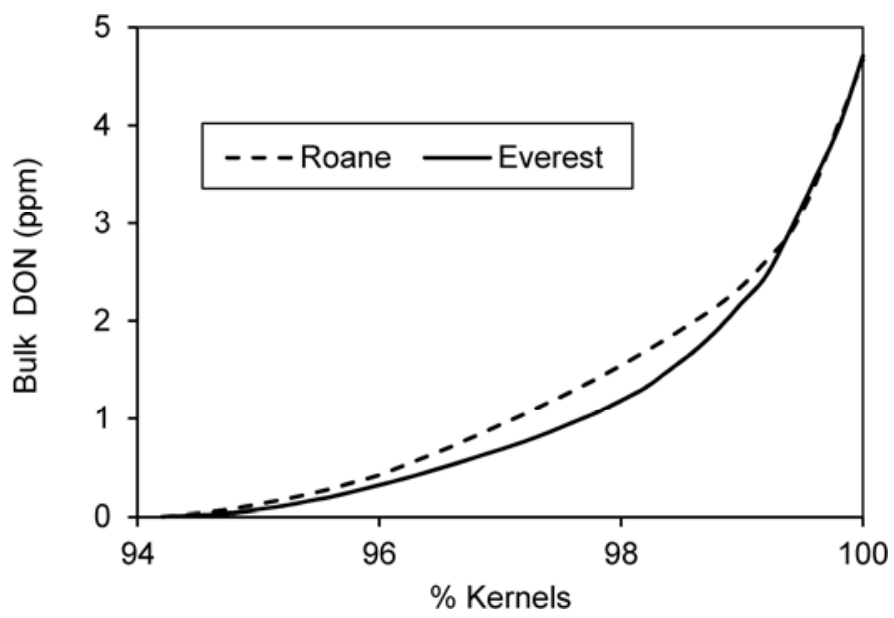

Fig. 6. Change in the cumulative bulk deoxynivalenol (DON) content of two wheat cultivars with 6.0\% DON-containing kernels (DCKs) and 4.7 ppm DON as the DCKs with the lowest to highest DON contents are added to sound kernels in each sample. it appears that Everest has a higher resistance to DON accumulation, such that a higher proportion of DCKs has a lower DON content compared with Roane. This finding may be because of the higher resistance of Everest to the accumulation of DON compared with Roane. Sneller et al (2012) showed that a significant genetic effect exists in wheat germplasm in terms of resistance to toxin accumulation, and these authors suggested that there is a need to understand this resistance to DON accumulation better and to develop rapid screening methods to assess this trait. Accordingly, the single-kernel DON analysis of grain samples may help to assess the resistance of wheat varieties for DON accumulation.

\section{CONCLUSIONS}

Our results showed that the bulk DON levels of small kernel samples can be estimated with the SKNIR method by evaluating the weight and predicted DON levels of single kernels. The single-kernel DON distribution among kernels can provide more insight into how DCKs contribute to the final DON levels of harvested grains. Two samples having the same DCKs and bulk DON levels can also be differentiated based on the DON distribution patterns among kernels, and the single-kernel DON distribution can be used to ascertain how variety resistance or fungicides reduce DON in harvested grain samples. Study of the distribution of single-kernel DON levels among kernels and among DCKs in a grain sample may be useful to evaluate varieties for their resistance to the spread of disease and their resistance to DON accumulation.

\section{LITERATURE CITED}

Dill-Macky, R. 2010. Fusarium head blight (scab). Pages 34-36 in: Compendium of Wheat Diseases and Pests. W. W. Bockus, R. L. Bowden, R. M. Hunger, W. L. Morrill, T. D. Murray, and R. W. Smiley, eds. American Phytopathological Society: St. Paul, MN.

Dowell, F. E., Maghirang, E. B., Graybosch, R. A., Baenziger, P. S., Baltensperger, D. D., and Hansen, L. E. 2006. An automated near-infrared system for selecting individual kernels based on specific quality characteristics. Cereal Chem. 83:537-543.

Finamore, A., Britti, M. S., Roselli, M., Bellovino, D., Gaetani, S., and Mengheri, E. 2004. Novel approach for food safety evaluation: Results of a pilot experiment to evaluate organic and conventional foods. J. Agric. Food Chem. 52:7425-7431.

Gilbert, J., and Woods, S. M. 2006. Strategies and considerations for multi-location FHB screening nurseries. In: The Global Fusarium Initiative for International Collaboration: A Strategic Planning Workshop Held at CIMMYT, El Batán, Mexico; March 14-17, 2006. T. Ban, J. M. Lewis, and E. E. Phipps, eds. CIMMYT: Mexico, D.F.

Jiang, G., Dong, Y., Lewis, J. M., Siler, L., and Ward, R. W. 2006. Characterization of resistance to Fusarium graminearum in a recombinant inbred line population of wheat: Resistance to fungal spread, mycotoxin accumulation, and grain yield loss and trait relationships. Crop Sci. 46:2590-2597.

Johnson, D. D., Wilson, W. W., and Diersen, M. A. 2001. Quality uncertainty, procurement strategies, and grain merchandising risk: Vomitoxin in spring wheat. Appl. Econ. Perspect. Pol. 23:102-119.

Koch, P. 2004. State of the art of trichothecenes analysis. Toxicol. Lett. 153:109-112.

Lemmens, M., Scholz, U., Berthiller, F., Asta, C. D., Koutnik, A., Schuhmacher, R., Adam, G., Buestmayr, H., Mesterhazy, A., Krska, R., and Ruckenbauer, P. 2005. The ability to detoxify the mycotoxin deoxynivalenol colocalizes with a major quantitative trait locus for Fusarium head blight resistance in wheat. Mol. Plant-Microbe Interact. 18:1318-1324.

Lilleboe, D. 2010. Fusarium head blight in 2010: An overview. Available at: http://scabusa.org/pdfs/USWBSI-Article_9-15-10.pdf

Lilleboe, D. 2011. Fusarium head blight in 2011: An overview. Available at: http://scabusa.org/pdfs/USWBSI-Article_2011-Update_9-29-11.pdf

McMullen, M., Jones, R., and Gallenberg, D. 1997. Scab of wheat and barley: A re-emerging disease of devastating impact. Plant Dis. 81:13401348. 
Mesterhazy, A. 1995. Types and components of resistance to Fusarium head blight. Plant Breed. 114:377-386.

Miedaner, T. 1997. Breeding wheat and rye for resistance to Fusarium diseases. Plant Breed. 116:201-220.

Mirocha, C. J., Kolaczkowski, E., Xie, W., Yu, H., and Jelen, H. 1998. Analysis of deoxynivalenol and its derivatives (batch and single kernel) using gas chromatography/mass spectrometry. J. Agric. Food Chem. 46:1414-1418.

Parry, D. W., Jenkinson, P., and McLeod, L. 1995. Fusarium ear blight (scab) in small cereals-A review. Plant Pathol. 44:207-238.

Peiris, K. H. S., Pumphrey, M. O., Dong, Y., Maghirang, E. B., Berzonsky, W., and Dowell, F. E. 2010. Near-infrared spectroscopic method for identification of Fusarium head blight damage and prediction of deoxynivalenol in single wheat kernels. Cereal Chem. 87:511-517.

Peiris, K. H. S., Pumphrey, M. O., Dong, Y., and Dowell, F. E. 2011.
Fusarium head blight symptoms and mycotoxin levels in single kernels of infected wheat spikes. Cereal Chem. 88:291-295.

Schroeder, H. W., and Christensen, J. J. 1963. Factors affecting the resistance of wheat to scab caused by Gibberella zeae. Phytopathology 53:831-838.

Sneller, C., Guttieri, M., Paul, P., Costa, J., and Jackwood, R. 2012. Variation for resistance to kernel infection and toxin accumulation in winter wheat infected with Fusarium graminearum. Phytopathology 102:306314.

Thompson, C. J. 2010. Evaluation of an inoculation method and quantitative trait loci for Fusarium head blight resistance in wheat. M.S. thesis. University of Illinois: Urbana-Champaign, IL.

Wegulo, S. N., and Dowell, F. E. 2008. Near-infrared versus visual sorting of Fusarium-damaged kernels in winter wheat. Can. J. Plant Sci. 88:1087-1089.

[Received November 26, 2012. Accepted August 6, 2013.] 